\title{
Análise cinemática da marcha pós protocolo de fadiga global de curta duração
}

\author{
Kinematic gait analysis post short-term global fatigue protocol \\ Leandro Viçosa Bonetti (D) 1,2 $₫$, Filipe Queiroz Triches (D) 1, Eduardo Carraro Armiliato (D) 1, Gabriel Johann Lazzari (D) 1 , \\ Vinícius Mazzochi Schmitt (D) 1, Fernanda Cechetti ${ }^{\mathbb{D}}{ }^{3}$, Raquel Saccani ${ }^{1}{ }^{1}$
}

1 Universidade de Caxias do Sul, Departamento de Fisioterapia. Caxias do Sul, RS, Brasil.

2 Universidade de Caxias do Sul, Programa de Pós-graduação em Ciências da Saúde. Caxias do Sul, RS, Brasil.

3 Universidade Federal de Ciências da Saúde de Porto Alegre, Programa de Pós-graduação em Ciências da Reabilitação. Porto Alegre, RS, Brasil.

Como citar este artigo (How to cite this article):

Bonetti LV, Triches FQ, Armiliato EC, Lazzari GJ, Schmitt VM, Cechetti F, Saccani R. Análise cinemática da marcha pós protocolo de fadiga global de curta duração (Kinematic gait analysis post short-term global fatigue protocol). Sci Med. 2019;29(4):e35217. https://doi.org/10.15448/1980-6108.2019.4.35217

\section{RESUMO}

OBJETIVO: Avaliar os efeitos de um protocolo de fadiga global de curta duração sobre os parâmetros cinemáticos da marcha de indivíduos saudáveis.

MÉTODOS: Fizeram parte da amostra 21 adultos jovens saudáveis. Foi realizada a análise de parâmetros da cinemática linear (velocidade, cadência, comprimento do passo, largura do passo e tempo do passo) e angular da marcha (flexão e extensão dos quadris; flexão e extensão dos joelhos; plantiflexão e dorsiflexão dos tornozelos) antes e após a realização de um protocolo de fadiga global de curta duração. Para a avaliação da marcha, foi utilizado um sistema de captura da trajetória tridimensional da marcha, composto por um sistema de cinemetria dotado de 7 câmeras integradas. O Protocolo de Fadiga de Agilidade Funcional de Curta Duração, que consiste em um protocolo de fadiga global de curta duração com séries de exercícios de agilidade funcional, foi utilizado para fadigar os participantes. Para as comparações entre as avaliações pré e pós-fadiga, os valores médios de cada avaliação foram utilizados e submetidos ao teste t pareado, e nível de significância adotado foi $\mathrm{p}<0,05$.

RESULTADOS: Não houveram diferenças estatisticamente significativas nos parâmetros angulares e lineares avaliados, exceto para cadência $(\mathrm{p}=0,03)$.

CONCLUSÕES: O protocolo de fadiga global de curta duração não alterou os parâmetros angulares e cinemáticos da marcha em indivíduos jovens, exceto a cadência que se mostrou mais elevada após o protocolo de fadiga.

DESCRITORES: Fadiga, marcha, fenômenos biomecânicos, voluntários saudáveis.

\section{ABSTRACT}

AIMS: Evaluate the effects of a short-term fatigue protocol on gait's kinematic parameters of healthy subjects.

METHODS: 21 healthy young adults participated in the sample. The parameters of linear kinematics of gait (velocity, cadence, step length, step width, step time) and angular kinematics of gait (hip's flexion and extension; knee's flexion and extension; ankle's dorsiflexion and plantar flexion) were analyzed before and after performing a short-term fatigue protocol. For gait evaluation, a three-dimensional gait trajectory capture system was used, consisting of a kinematic system with 7 integrated cameras. The Short-Term Functional Agility Fatigue Protocol, which consists of a short-term global fatigue protocol with series of functional agility exercises, was used to fatigue participants. For comparisons between pre and post fatigue evaluations, the mean values to paired $\mathrm{t}$ test, and statistical significance was set as $\mathrm{p}<0.05$.

RESULTS: There were no statistically significant differences in the angular and linear parameters evaluated, except for cadence $(p=0.03)$. CONCLUSIONS: The short-term global fatigue protocol did not alter the angular and kinematic gait parameters in young subjects, except for the cadence, that was higher after the fatigue protocol.

KEYWORDS: Fatigue, gait, biomechanical phenomena, healthy volunteers. 


\section{INTRODUÇÃO}

A fadiga muscular é considerada um fenômeno comum durante e após a prática esportiva ou prática de alguma atividade física [1]. Estudos clássicos definem a fadiga muscular como uma redução da capacidade de um músculo, ou grupo muscular, produzir ou manter os níveis normais de força e/ou potência $[2,3]$. Essa redução das capacidades musculares é dependente da quantidade, intensidade e volume; porém é independente do tipo da atividade física ou do exercício físico. De uma forma geral, os protocolos de exercícios físicos utilizados para o estudo da fadiga muscular são divididos em exercícios musculares locais, que se concentram em exercitar um músculo ou grupo muscular em específico; e em exercícios musculares gerais, que mobilizam todo o corpo durante a atividade [4].

Devido a fadiga muscular estar relacionada a um aumento no risco de quedas durante a marcha e a marcha ser considerada um importante indicador sobre a saúde geral das pessoas, esse tema é de grande interesse por parte da literatura científica $[5,6]$. Entretanto, as heterogêneas metodologias, tanto na escolha do protocolo de fadiga quanto na análise dos dados da marcha, não permitem um consenso sobre os reais efeitos da fadiga sobre os parâmetros da marcha. Alguns estudos investigaram as alterações da marcha após a fadiga de grupos musculares específicos, como quadríceps [7-11] e isquiotibiais [10,11]. No entanto, os protocolos de fadiga muscular localizada permitem interpretações limitadas de seus resultados pois utilizam atividades com foco muscular local, e por não se tratarem de atividades que envolvam atividades de vida diária, não reproduzem situações reais de fadiga muscular [12].

Adicionalmente, protocolos de fadiga global também foram utilizados para verificar sua influência na marcha. Estes protocolos mobilizam uma grande quantidade de músculos e têm um impacto maior no sistema muscular [13]. Estes estão relacionados às atividades de vida diária, às atividades ocupacionais ou até mesmo às atividades físicas; como o teste de caminhada de seis minutos [14], a corrida em esteira ergométrica [15,16], utilização do ciclo ergômetro [17] e inclusive a realização das atividades de levantar e sentar em uma cadeira $[18,19]$ ou agachamento [5]. Porém, segundo Vieira et al., ainda não há consenso científico sobre as alterações nos parâmetros da marcha após um protocolo de fadiga global [16]. Além disso, as alterações biomecânicas após a realização de protocolos de fadiga global de curta duração foram observadas através de movimentos específicos como saltos e marcha lateral; e não através da análise da marcha $[20,21]$. Deste modo, o objetivo deste estudo foi avaliar os efeitos de um protocolo de fadiga global de curta duração sobre os parâmetros cinemáticos da marcha de indivíduos jovens saudáveis.

\section{MÉTODOS}

O presente estudo se caracteriza por um estudo descritivo, com delineamento transversal [22]. Fizeram parte da amostra, 21 adultos jovens saudáveis, sem alterações neuromusculoesqueléticas e com idade inferior a 30 anos. O número de participantes foi estabelecido de forma intencional e não probabilística, de acordo com o número de sujeitos disponíveis para a participação na pesquisa [22]. Fizeram parte dos critérios de inclusão: a) participantes entre 18 e 30 anos de idade; b) assinatura do Termo de Consentimento Livre e Esclarecido; c) participantes sem lesões neuromusculoesqueléticas que dificultassem a realização do protocolo de fadiga global de curta duração ou a avaliação da marcha. Os critérios de exclusão foram: a) impossibilidade de conclusão do protocolo de fadiga; b) impossibilidade de conclusão das avaliações da marcha, como por lesão aguda; c) realização de atividade física nas 48 horas prévias à coleta de dados; d) presença de doenças cardiovasculares não controladas; e) participantes do sexo feminino que estivessem em período gestacional; f) erro durante a coleta de dados.

Este estudo foi aprovado (parecer 2.230.696) pelo Comitê de Ética e Pesquisa da Universidade Federal de Ciências da Saúde de Porto Alegre e conduzido de acordo com as disposições legais da resolução no 466 do ano de 2012, do Conselho Nacional de Saúde, que aprova as diretrizes e normas regulamentadoras de pesquisas envolvendo seres humanos. Todos os testes foram realizados no Laboratório de Marcha do Centro Clínico da Universidade de Caxias do Sul.

Para a coleta de dados, os participantes se dirigiram ao laboratório de marcha, receberam explicações sobre a pesquisa, sobre os procedimentos e assinaram o Termo de Consentimento Livre e Esclarecido. Em caso de dúvidas, estas foram sanadas pelos pesquisadores. Em seguida, um questionário sobre identificação pessoal, massa corporal, estatura, índice de massa corporal, estado de saúde, nível de atividade física e lesões neuromusculoesqueléticas foi aplicado. Para verificar o nível de atividade física, foi utilizado o Questionário Internacional de Atividade Física (International 
Physical Activity Questionnaire). Esta ferramenta é amplamente utilizada em diversos países, tendo sido validada em diferentes idiomas [23,24]. Os indivíduos foram classificados em: (1) sedentários (não realizaram nenhuma atividade física por pelo menos 10 minutos contínuos durante a semana); (2) insuficientemente ativos (realizaram atividades físicas por pelo menos 10 minutos contínuos durante a semana, porém de maneira insuficiente para ser classificado como ativos); (3) ativos (cumpriram as seguintes recomendações: a) atividade física vigorosa $-\geq$ três dias/semana e $\geq 20 \mathrm{~min} / \mathrm{sessão}$; b) moderada ou caminhada $-\geq$ cinco dias/semana e $\geq 30 \mathrm{~min} / \mathrm{sessão}$; c) qualquer atividade somada: $\geq$ cinco dias/semana e $\geq 150 \mathrm{~min} /$ semana); (4) muito ativos (cumpriram as seguintes recomendações: a) vigorosa $-\geq$ cinco dias/semana e $\geq 30 \mathrm{~min} / \mathrm{sessão}$; b) vigorosa $-\geq$ três dias/semana e $\geq 20 \mathrm{~min} / \mathrm{sessão}$ + moderada e ou caminhada $\geq$ cinco dias/semana e $\geq 30 \mathrm{~min} /$ sessão).

Os procedimentos de avaliação da marcha pré fadiga seguiram o protocolo de Laroche et al. [25]. Para adaptação ao protocolo de marcha, foi solicitado aos participantes que caminhassem oito metros em linha reta, no local destinado à coleta de dados da marcha, em uma velocidade habitual. Os participantes memorizaram o número de passos e o ritmo necessário para serem capazes de realizar o contato com as duas plataformas de força, ora com o pé direito inteiro, ora com o pé esquerdo. Após a familiarização, foram afixados marcadores reflexivos (Figura 1), seguindo o protocolo do sistema e VICON MX systems [26] nos seguintes pontos anatômicos a direita e esquerda: espinha ilíaca ântero-superior, espinha ilíaca pósterosuperior, porção médio-lateral do fêmur, porção medial e lateral do joelho, porção médio-lateral da tíbia, porção
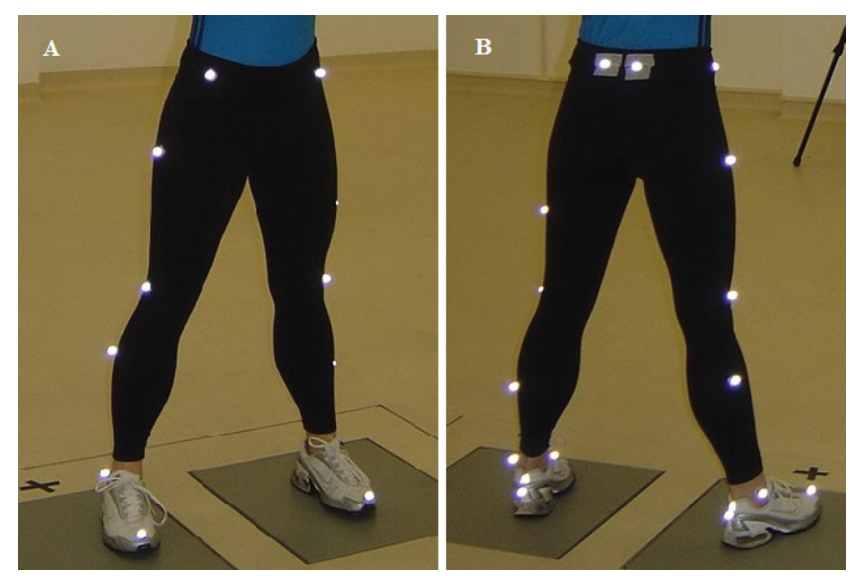

Figura 1. Ilustração dos pontos anatômicos onde foram colocados os marcadores reflexivos de acordo com o protocolo do com o VICON MX systems [26]. $\mathrm{A}=$ vista anterior; $\mathrm{B}=$ vista posterior. medial e lateral do tornozelo, porção centro-posterior do calcâneo e face dorsal do segundo metatarso. O protocolo de avaliação consistiu na realização da marcha de modo que os participantes passassem pelas duas plataformas, sendo necessária a captação de oito passos válidos. Não houve limite de tentativas [25].

Após a avaliação da marcha pré-fadiga, os participantes realizaram o protocolo de fadiga global de curta duração adaptado de Cortes et al. [21]. O protocolo consiste na realização de exercícios em blocos ou séries. Primeiramente, um salto horizontal máximo foi realizado. Este teste foi realizado como o parâmetro de fadiga dos participantes, considerando estes fadigados quando repetiram o salto e não atingiram $90 \%$ ou mais do valor do primeiro salto. Após o primeiro salto horizontal, os participantes iniciaram o protocolo de fadiga, que consistiu em uma série de exercícios: (1) seis saltos verticais máximos; (2) duas séries de corridas de cinco, 10 e cinco metros em velocidade máxima e sem intervalos; (3) seis agachamentos livres com $90^{\circ}$ de flexão de joelho; e (4) subida e descida de um step por 40 segundos. Os participantes foram orientados a realizar os exercícios com o máximo de suas capacidades físicas e sem pausas. Após a realização de cada série, ou se a série fosse interrompida por alegação de fadiga ou cansaço, um salto horizontal era novamente realizado para verificar se o participante estava fadigado. Se o participante atingiu um valor igual ou maior a $90 \%$ do primeiro salto horizontal, uma nova série dos exercícios era recomeçada ou continuava a série interrompida anteriormente; mas se era inferior à $90 \%$, o protocolo dos exercícios era interrompido e o participante era encaminhado para a avaliação de marcha pós fadiga.

As variáveis coletadas foram tabuladas e analisadas através do programa IBM SPSS Statistics 21. As variáveis cinemáticas lineares analisadas foram: a) velocidade e cadência (inferência espaço-temporal); b) comprimento do passo e largura do passo (inferência espacial); c) tempo do passo (inferência temporal); enquanto as variáveis cinemáticas angulares foram: a) flexão e extensão dos quadris; b) flexão e extensão dos joelhos; c) plantiflexão e dorsiflexão dos tornozelos. Utilizou-se a estatística descritiva para sumarizar os dados, os quais são apresentados como média e desviopadrão (medidas de tendência central e variabilidade) ou distribuição de frequência. Para as comparações das variáveis quantitativas entre as avaliações pré e pós fadiga foi aplicado o teste de normalidade (Shapiro Wilk) e, devido à distribuição paramétrica dos dados, foi adotado o teste $t$ pareado, considerando nível de significância de $5 \%$. 


\section{RESULTADOS}

A Tabela 1 apresenta a característica antropométrica e o nível de atividade física da amostra. Com relação ao

Tabela 1. Características dos participantes

\begin{tabular}{lccc}
\hline Variáveis & Média \pm DP & Mínimo & Máximo \\
\hline Idade (anos) & $23,14 \pm 3,04$ & 18 & 29 \\
Massa corporal $(\mathrm{kg})$ & $71,97 \pm 16,60$ & 51,70 & 110,10 \\
Estatura $(\mathrm{m})$ & $1,70 \pm 0,91$ & 1,57 & 1,88 \\
Índice de massa corporal $\left(\mathrm{kg} / \mathrm{m}^{2}\right)$ & $24,78 \pm 3,96$ & 20,30 & 36,00 \\
Nível de atividade física & $\mathrm{n}$ & & \\
Sedentários & 0 & & \\
Insuficientemente ativos & 12 & & \\
Ativos & 9 & & \\
Muito ativos & 0 & & \\
Fadiga & Média & & \\
Tempo para fadiga $(\mathrm{min})$ & 6,25 & \\
\hline
\end{tabular}

$\mathrm{DP}=$ desvio padrão; $\mathrm{kg}=$ quilogramas; $\mathrm{m}=$ metros; $\mathrm{kg} / \mathrm{m}^{2}=$ quilograma por metro quadrado; $\min =$ minutos; $\mathrm{n}=$ número de participantes. índice de massa corporal, o valor médio dos indivíduos foi de $24,78( \pm 3,96) \mathrm{kg} / \mathrm{m}^{2}$, valor considerado normal segundo a Organização Mundial de Saúde [27]. Entretanto, dois participantes apresentaram valores considerados como sobrepeso enquanto quatro como obesidade. Com relação aos níveis de atividade física, de acordo com os resultados do Questionário Internacional de Atividade Física, 12 participantes foram considerados insuficientemente ativos enquanto nove foram considerados ativos.

Na Tabela 2 são apresentados os valores médios da cinemática linear da marcha pré e pós protocolo de fadiga. Nessa comparação, apenas o parâmetro cadência apresentou diferença estatisticamente significativa $(p=0,03)$, com o valor da avaliação pós sendo maior quando comparado ao valor pré fadiga. Já a Tabela 3 apresenta os valores médios da cinemática angulares da marcha pré e pós fadiga. Nenhuma diferença estatisticamente significativa foi verificada, em nenhuma articulação estudada.

Tabela 2. Média \pm DP das variáveis cinemáticas lineares da marcha, pré e pós a realização de um protocolo de fadiga global de curta duração.

\begin{tabular}{|c|c|c|c|c|c|c|}
\hline Variáveis lineares & \multicolumn{2}{|c|}{ Pré fadiga } & \multicolumn{2}{|c|}{ Pós fadiga } & \multicolumn{2}{|c|}{ p-valor } \\
\hline Velocidade $(\mathrm{m} / \mathrm{s})$ & \multicolumn{2}{|c|}{$1,29 \pm 0,15$} & \multicolumn{2}{|c|}{$1,34 \pm 0,16$} & \multicolumn{2}{|c|}{0,06} \\
\hline \multirow[t]{2}{*}{ Cadência (p/min) } & \multicolumn{2}{|c|}{$114,65 \pm 9,57$} & \multicolumn{2}{|c|}{$116,80 \pm 11,47$} & \multicolumn{2}{|c|}{0,03} \\
\hline & direita & esquerda & direita & esquerda & direita & esquerda \\
\hline Comprimento do passo $(\mathrm{m})$ & $0,68 \pm 0,05$ & $0,68 \pm 0,05$ & $0,68 \pm 0,04$ & $0,68 \pm 0,05$ & 0,72 & 0,48 \\
\hline Largura do passo (m) & $0,15 \pm 0,02$ & $0,15 \pm 0,02$ & $0,16 \pm 0,02$ & $0,16 \pm 0,03$ & 0,09 & 0,10 \\
\hline Tempo do passo (s) & $0,53 \pm 0,04$ & $0,52 \pm 0,05$ & $0,52 \pm 0,05$ & $0,52 \pm 0,05$ & 0,40 & 0,89 \\
\hline
\end{tabular}

$\mathrm{DP}=$ desvio padrão; $\mathrm{m} / \mathrm{s}=$ metros por segundo; $\mathrm{p} / \mathrm{min}=$ passos por minuto; $\mathrm{s}=$ segundos; $\mathrm{m}=$ metros.

Tabela 3. Média e desvio-padrão das variáveis cinemáticas angulares da marcha, pré e pós a realização de um protocolo de fadiga global de curta duração.

\begin{tabular}{lccc}
\hline Variáveis angulares (o) & Pré fadiga & Pós fadiga & p-valor \\
Quadril & & & \\
$\quad$ Flexão D & $36,41 \pm 8,19$ & $36,20 \pm 6,31$ & 0,80 \\
$\quad$ Flexão E & $37,23 \pm 7,92$ & $36,41 \pm 6,30$ & 0,29 \\
$\quad$ Extensão D & $-7,66 \pm 8,61$ & $-7,70 \pm 7,49$ & 0,97 \\
Extensão E & $-7,81 \pm 8,11$ & $-8,70 \pm 6,43$ & 0,32 \\
Joelho & & & \\
Flexão D & $60,40 \pm 8,92$ & $60,48 \pm 8,60$ & 0,90 \\
Flexão E & $60,08 \pm 9,42$ & $59,58 \pm 9,10$ & 0,80 \\
Extensão D & $1,13 \pm 6,34$ & $1,20 \pm 6,52$ & 0,94 \\
Extensão E & $1,06 \pm 5,44$ & $1,20 \pm 6,04$ & 0,85 \\
Tornozelo & & & \\
Plantiflexão D & $-14,76 \pm 8,73$ & $-17,48 \pm 5,50$ & 0,07 \\
Plantiflexão E & $-16,31 \pm 3,62$ & $-17,38 \pm 5,88$ & 0,21 \\
Dorsiflexão D & $12,90 \pm 6,42$ & $13,05 \pm 3,31$ & 0,90 \\
Dorsiflexão E & $13,88 \pm 2,47$ & $13,63 \pm 3,76$ & 0,69 \\
\hline
\end{tabular}

Valores expressos em graus.

\section{DISCUSSÃO}

O presente estudo avaliou os efeitos de um protocolo de fadiga global de curta duração sobre as variáveis cinemáticas lineares e angulares da marcha de indivíduos jovens saudáveis. Os protocolos globais de curta duração são protocolos que utilizam movimentos e habilidades atléticas; e são interessantes para a avaliações biomecânicas devido à associação entre a fadiga muscular e suas consequências [28]. Os resultados deste estudo demonstraram que, com exceção da variável cadência, o protocolo de fadiga utilizado não altera significativamente a marcha de sujeitos saudáveis.

O protocolo de fadiga global de curta duração não alterou significativamente nenhuma das variáveis cinemáticas angulares da marcha analisadas neste estudo. Contraditoriamente, outros protocolos de fadiga global alteraram alguns parâmetros angulares 
em jovens saudáveis. McLoughlin et al. observaram que durante a fase de apoio da marcha ocorreram aumentos na flexão máxima do quadril e do joelho, além de aumentos na extensão máxima do joelho, na dorsiflexão e plantiflexão máxima do tornozelo [14]; de forma similar, Qu e Yeo demonstraram maiores amplitudes de movimento das articulações do quadril e joelho [15]. Após a fadiga do quadríceps, Parijat e Lockhart relataram redução da extensão e aumento da flexão máxima do joelho [7]; porém Murdock e HubleyKozey não observaram alterações significativas [8]. A fadiga do quadríceps e dos isquiotibiais também não alteraram os ângulos de flexão do joelho, mas a extensão máxima do joelho foi reduzida [11].

No que diz respeito às variáveis cinemáticas lineares da marcha; o comprimento, tempo e largura do passo não apresentaram diferenças estatisticamente significativas na comparação pré/pós fadiga. Estudos prévios demonstraram que protocolos de fadiga de músculos específicos dos membros inferiores e protocolos de fadiga global também não foram suficientes para alterar o comprimento do passo ou passada $[5,9,11,16,19]$. No que se refere ao tempo do passo, o exercício de levantar e sentar de uma cadeira também não teve influência sobre este parâmetro $[18,19]$. No entanto, o exercício de levantar e sentar [18] e o agachamento [5] aumentaram significativamente a largura do passo, diferentemente dos resultados da presente pesquisa.

Entretanto, os dois parâmetros da cinemática linear mais comumente estudados são os parâmetros espaçotemporais, a velocidade e a cadência. O protocolo de fadiga não alterou significativamente a velocidade da marcha. Este resultado também foi encontrado em adultos jovens após a fadiga de grupos musculares específicos, como quadríceps [7-9] e quadríceps e isquiotibiais [11]; assim como após os protocolos de fadiga global que utilizaram o ciclo ergômetro e exercícios de agachamento [5,17]. Entretanto, o exercício de sentar e levantar e o teste de caminhada de seis minutos realizado em velocidade máxima aumentaram significativamente a velocidade da marcha $[14,18]$. Já a cadência, definida como o número de passos dados em um determinado período de tempo, foi o único parâmetro do presente estudo influenciado significativamente pelo protocolo de fadiga global de curta duração [29]. Os participantes apresentaram um aumento significativo no número de passos por minuto pós fadiga $(p=0,03)$. Apesar de serem protocolos diferentes do protocolo utilizado na presente pesquisa, os protocolos de fadiga global de McLoughlin et al. e Arif et al. também aumentaram significativamente a cadência de adultos saudáveis $[14,17]$.
Referente ao protocolo de fadiga global de curta duração utilizado ter alterado significativamente apenas a cadência da marcha, alguns fatores devem ser considerados. Primeiramente, para Granacher et al., o desconforto físico provocado pela fadiga pode levar os participantes a aumentarem a velocidade ou a cadência da marcha com o intuito de finalizar a avaliação o mais breve possível [10]. Ademais, Cortes et al. relatam que o decréscimo dos valores do salto horizontal sugere que este protocolo efetivamente induziu os mesmos níveis de fadiga muscular de protocolos de longa duração $[20,21]$. Além disso, o tempo médio para atingir a fadiga na presente pesquisa foi de aproximadamente 6 minutos e 15 segundos (6,25 min), valor idêntico aos seis minutos relatados por Cortes et al. [20]. Por isso, a idade parece ser a principal causa da manutenção de um padrão cinemático da marcha mesmo fadigados. Adultos jovens saudáveis têm uma grande capacidade de manter uma marcha normal, mesmo fadigados $[17,19]$.

Alguns estudos demonstraram que alterações em parâmetros da marcha após a fadiga têm o objetivo compensatório de manter a estabilidade durante a locomoção [5]. O exemplo mais comum ocorre quando a largura do passo ou da passada aumenta com o objetivo de aumentar a base de suporte do corpo $[30,31]$. Entretanto, pode-se observar que a literatura apresenta resultados controversos sobre as alterações da marcha após a fadiga muscular. Este fato ocorre devido à heterogeneidade metodológica utilizada na coleta e análise dos dados e aos diferentes protocolos de fadiga afetarem de forma variada a musculatura dos membros inferiores $[15,20]$.

No entanto, esta pesquisa apresentou algumas limitações, como ausência de outras análises angulares dos membros inferiores e análises em plano frontal; ausência de participantes de outras faixas etárias ou participantes de grupos patológicos; heterogeneidade e seleção da amostra ter ocorrido de forma intencional e não probabilística.

Conclui-se que o protocolo de fadiga global de curta duração utilizado não alterou a maioria dos parâmetros cinemáticos lineares e angulares da marcha. Apesar de estarem comprovadamente fadigados, a manutenção de um padrão cinético pós fadiga parece estar relacionado à idade destes indivíduos, demonstrando que adultos jovens têm grande capacidade em manter a marcha normal mesmo fadigados.

\section{NOTAS}

\section{Apoio financeiro}

Este estudo não recebeu apoio financeiro de fontes externas. 


\section{Declaração de conflito de interesses}

Os autores declaram não haver conflitos de interesses relevantes ao conteúdo deste estudo.

\section{Contribuições dos autores}

Todos os autores fizeram contribuições substanciais para concepção, ou delineamento, ou aquisição, ou análise ou inter- pretação de dados; e redação do trabalho ou revisão crítica; e aprovação final da versão para publicação.

Disponibilidade dos dados e responsabilidade pelos resultados

Todos os autores declaram ter tido total acesso aos dados obtidos e assumem completa responsabilidade pela integridade destes resultados.

\section{REFERÊNCIAS}

1. Rimmer JH, Schiller W, Chen MD. Effects of disability-associated low energy expenditure deconditioning syndrome. Exerc Sport Sci Ver. 2012;40(1):22-9. https://doi.org/10.1097/jes.0b013e31823b8b82

2. Søgaard K, Gandevia SC, Todd G, Petersen NT, Taylor JL. The effect of sustained low-intensity contractions on supraspinal fatigue in human elbow flexor muscles. J Physiol. 2006;573(2):511-23. https://doi.org/10.1113/jphysiol. 2005.103598

3. Enoka RM, Duchateau J. Muscle fatigue: what, why and how it influences muscle function. J Physiol. 2008;586(1):11-23. https://doi.org/10.1113/jphysiol.2007.139477

4. Paillard T. Effects of general and local fatigue on postural control: a review. Neurosci Biobehav Rev. 2012;36(1): 162-76.

5. Zhang J, Lockhart TE, Soangra R. Classifying lower extremity muscle fatigue during walking using machine learning and inertial sensors. Ann Biomed Eng. 2014;42(3):600-12. https://doi.org/10.1007/s10439-013-0917-0

6. Cho YS, Jang SH, ChoJS, Kim MJ, Lee HD, Lee SY, et al. Evaluation of validity and reliability of inertial measurement unit-based gait analysis systems. Ann Rehabil Med. 2018;42(6):872-83. https://doi.org/10.5535/arm. 2018.42.6.872

7. Parijat P, Lockhart TE. Effects of quadriceps fatigue on the biomechanics of gait and slip propensity. Gait Posture. 2008;28(4):568-73. https://doi.org/10.1016/j.gaitpost.2008.04.001

8. Murdock G, Hubley-Kozey C. Effect of a high intensity quadriceps fatigue protocol on knee joint mechanics and muscle activation during gait in young adults. Eur J Appl Physiol. 2012;112(2):439-49. https://doi.org/10.1007/ s00421-011-1990-4

9. Barbieri FA, Beretta SS, Pereira VA, Simieli L, Orcioli-Silva D, dos Santos PCR, van Dieën JH, Gobbi LT. Recovery of gait after quadriceps muscle fatigue. Gait Posture. 2016;43(1):270-4. https://doi.org/10.1016/j.gaitpost. 2015.10.015

10. Granacher U, Wolf I, Wehrle A, Bridenbaugh S, Kressig RW. Effects of muscle fatigue on gait characteristics under single and dual-task conditions in young and older adults. J Neuroeng Rehabil. 2010;7(1):56-67. https://doi. org/10.1186/1743-0003-7-56

11. Longpré HS, Potvin JR, Maly MR. Biomechanical changes at the knee after lower limb fatigue in healthy young women. Clin Biomech. 2013;28(4):441-7. https://doi.org/10.1016/j.clinbiomech.2013.02.010

12. Barbieri FA, Gobbi LTB, Lee YJ, Pijnappels M, van Dieën JH. Effect of triceps surae and quadriceps muscle fatigue on the mechanics of landing in stepping down in ongoing gait. Ergonomics. 2014;57(6):934-42. https://doi.org/ $10.1080 / 00140139.2014 .903302$

13. Fox ZG, Mihalik JP, Blackburn JT, Battaglini CL, Guskiewicz KM. Return of postural control to baseline after anaerobic and aerobic exercise protocols. J Athl Train. 2008;43(5):456-63.

https://doi.org/10.4085/1062-6050-43.5.456

14. McLoughlin JV, Barr CJ, Patritti B, Crotty M, Lord SR, Sturnieks DL. Fatigue induced changes to kinematic and kinetic gait parameters following six minutes of walking in people with multiple sclerosis. Disabil Rehabil. 2016;38(6):535-43. https://doi.org/10.3109/09638288.2015.1047969

15. Qu X, Yeo JC. Effects of load carriage and fatigue on gait characteristics. J Biomech. 2011;44(7):1259-63. https://doi.org/10.1016/j.jbiomech.2011.02.016 
16. Vieira MF, e Souza GSDS, Lehnen GC, Rodrigues FB, Andrade AO. Effects of general fatigue induced by incremental maximal exercise test on gait stability and variability of healthy young subjects. J Electromyogr Kinesiol. 2016;30(1): 161-7. https://doi.org/10.1016/j.jelekin.2016.07.007

17. Arif M, Ohtaki Y, Nagatomi R, Inooka H. Analysis of the effect of fatigue on walking gait using acceleration sensor placed on the waist. Eng Intell Syst Elec. 2010;18(2):93-103.

18. Barbieri FA, dos Santos PCR, Vitório R, van Dieën JH, Gobbi LTB. Effect of muscle fatigue and physical activity level in motor control of the gait of young adults. Gait Posture. 2013;38(4):702-07. https://doi.org/10.1016/j. gaitpost.2013.03.006

19. Santos PCR, Gobbi LTB, Orcioli-Silva D, Simieli L, van Dieën JH, Barbieri FA. Effects of leg muscle fatigue on gait in patients with Parkinson's disease and controls with high and low levels of daily physical activity. Gait Posture. 2016;47(1):86-91. https://doi.org/10.1016/j.gaitpost.2016.04.002

20. Cortes N, Quammen D, Lucci S, Greska E, Onate J. A functional agility short-term fatigue protocol changes lower extremity mechanics. J Sports Sci. 2012;30(8):797-805. https://doi.org/10.1080/02640414.2012.671528

21. Cortes N, Greska E, Ambegaonkar RKJ, Onate JA. Changes in lower extremity biomechanics due to a short-term fatigue protocol. J Athl Train. 2013;3(48):306-13. https://doi.org/10.4085/1062-6050-48.2.03

22. Thomas JR, Nelson JK. Métodos de pesquisa em atividade física. Porto Alegre: Artmed, 2007.

23. Hagströmer M, Oja P, Sjöström M. The International Physical Activity Questionnaire (IPAQ): a study of concurrent and construct validity. Public Health Nutr. 2006;9(6):755-62. https://doi.org/10.1079/phn2005898

24. Wanner M, Probst-Hensch N, Kriemler S, Meier F, Autenrieth C, Martin BW. Validation of the long international physical activity questionnaire: influence of age and language region. Prev Med Rep. 2016;3(1):250-6. https://doi. org/10.1016/j.pmedr.2016.03.003

25. Laroche D, Duval A, Morisset C, Beis JN, D'athis P, Maillefert JF, Ornetti P. Test-retest reliability of 3D kinematic gait variables in hip osteoarthritis patients. Osteoart Cart. 2011;19(2):194-9. https://doi.org/10.1016/j.joca.2010.10.024

26. VICON MX systems. Vicon Documentation. Lower body modeling with Plug-in Gait [cited 19 Oct 2019]. Avalaible from: https://docs.vicon.com/display/Nexus25/Lower+body+modeling+with+Plug-in+Gait. https://doi.org/10.1016/j. gaitpost.2011.10.312

27. Global Health Observatory data repository. Prevalence of overweight among adults, BMI $\geq 25$, age-standardized. Estimates by country. Geneva: WORLD HEALTH ORGANIZATION (Organização Mundial de Saúde - OMS); 2017. [cited 2019 Jul 15]. Avalaible from: http://apps.who.int/gho/data/node.main.A897A?lang=en

28. Grassi B, Rossiter HB, Zoladz JA. Skeletal muscle fatigue and decreased efficiency: two sides of the same coin?. Exerc Sport Sci Rev. 2015;43(2):75-83. https://doi.org/10.1249/jes.0000000000000043

29. Stansfield B, Clarke C, Dall P, Godwin J, Holdsworth R, Granat M. True cadence and step accumulation are not equivalent: The effect of intermittent claudication on free-living cadence. Gait Posture. 2015;41(2):414-9. https://doi. org/10.1016/j.gaitpost.2014.11.002

30. Nordin E, Moe-Nilssen R, Ramnemark A, Lundin-Olsson L. Changes in step-width during dual-task walking predicts falls. Gait Posture. 2010;32(1):92-7. https://doi.org/10.1016/j.gaitpost.2010.03.012

31. Grabiner MD, Marone JR, Wyatt M, Sessoms P, Kaufman KR. Performance of an attention-demanding task during treadmill walking shifts the noise qualities of step-to-step variation in step width. Gait Posture. 2018;63(1):154-8. https://doi.org/10.1016/j.gaitpost.2018.04.041 